\title{
EL, VERBO DE LAS ORACIONES ESCINDIDAS: IMPLICACIONES TEÓRICAS
}

\begin{abstract}
A bstract. Linde-Usiekniewicz Jadwiga, El verbo de las oraciones escindidas: implicaciones teóricas [The verb of cleft sentences: theorctical implications]. Studia Romanica Posnaniensia, Adam Mickiewicz University Press, Poznań, vol. XXXI: 2004, pp. 321-327. ISBN 83-232-1353-4, ISSN 0137-2475.
\end{abstract}

The paper discusses some possible implications arising from the fact that in Spanish all kinds of clcft sentences are apparently pseudo-clefts and both the linking verb and the verb of the relative clause may agree with the clefted subject and possibly object.

En el presente artículo intentaremos discutir ciertas implicaciones teóricas que surgen de las formas personales que adoptan los verbos en las oraciones escindidas. Nos referimos tanto al verbo copulativo que constituye oraciones tales como el nexo oracional de primer rango, como a la forma del verbo original, el cual aparece en la oración no escindida correspondiente.

En la literatura linguística el problema de las oraciones escindidas se ha discutido ampliamente dentro del marco de la gramática generativa. Para nuestros fines es suficiente recordar dos de sus características básicas.

La primera es que por su estructura sintáctica las oraciones escindidas españolas se parecen mucho a las oraciones compuestas con una oración subordinada de relativo, e.g.

\section{Fue de Juan de quien te hablé.}

De ahí su nombre español tradicional de relativas perifrásticas (Fernández Ramirez 1951). La segunda es la función comunicativa de tales oraciones: en ellas la división del enunciado en tópico y foco queda inequívocamente marcada de tal manera que el contenido de la relativa es siempre tópico y el elemento escindido es el foco (Moreno Cabrera 1999).

Ahora bien, las oraciones escindidas españolas tienen ciertos rasgos particulares, por los cuales difieren de sus correspondientes en algunas otras lenguas. Estos 
son, primero, el hecho de que en las escindidas llamadas focalizantes, en las cuales el elemento escindido precede a la relativa, éste no lleva ningún pronombre (o proforma) inicial. Segundo, desde el punto de vista de su estructura sintáctica, las escindidas topicalizantes y focalizantes aparentemente difieren sólo en cuanto al orden:

\section{Fue de Juan de quien te hablé.}

3. De quien te hablé fue de Juan.

Se podría argumentar que la semejanza superficial entre las oraciones focalizantes y topicalizantes, o sea entre las de tipo (2) y las de tipo (3), es solamente superficial y que en realidad representan oraciones compuestas diferentes. No cabe duda que las topicalizantes (3) son trimembres y están compuestas de elemento copulativo y de dos argumentos de la cópula: el pre-copular y relativo y el post-copular escindido. La oración relativa es una relativa libre, o sea sin antecedente.

Ahora las focalizantes a primera vista parecen estar construidas de manera diferente. Por su orden se asemejan a las oraciones compuestas con una relativa atributiva, que modificaría entonces al elemento escindido. Esta semejanza es aun más notable cuando lo que vuelve a ser el elemento escindido es el sujeto de la oración original, o sea:

\section{Fue Juan quien lo hizo.}

donde se podría postular una estructura bimembre: elemento copulativo y su argumento único, compuesto del sustantivo antecedente de la relativa y la relativa misma. Tal relativa tendría forzosamente que ser una relativa explicativa por las siguientes razones. Primero, es una relativa que puede modificar a un nombre propio que normalmente no acepta la especificación. Segundo, en términos de entonación, hay posibilidad de introducir una pausa entre el supuesto antecedente y la relativa, lo que es propio de las relativas explicativas.

Pero el análisis en tales términos esta lleno de fallos. En primer lugar, la relativa de este tipo, incluso siendo separada por pausa, nunca lleva una entonación de tipo parentético. Por tanto es difícil interpretarla como explicativa. En segundo lugar, los nexos relativos que en ella aparecen no son propios de oraciones explicativas, sino de oraciones especificativas sin antecedente. En tercer lugar, el hecho de construirse la parte post-copular de tales oraciones como un sólo elemento sintáctico constituiría un desajuste con el papel funcional de tales oraciones, cuya función principal es marcar claramente la división entre el tópico y el foco. $\mathrm{Si}$ adoptamos el análisis con relativa explicativa, tenemos al tópico modificando al foco, lo que es contrario a todo lo que se puede esperar.

Hasta este momento nos hemos referido a los dos elementos nominales unidos por la cópula como pre- y post-copulares, sin entrar en los pormenores del tipo de oración compuesta que es la oración escindida. Del hecho de que el elemento 
constituyente de tales oraciones sea el verbo ser se puede concluir que se trata de un subtipo de oraciones de predicado nominal.

Efectivamente, las oraciones escindidas son desde el punto de vista estructural y lógico oraciones identificativas o de identidad, en las cuales se consta la equivalencia referencial del contenido del elemento escindido y de la relativa. El único argumento posible en contra de tal análisis es la existencia de oraciones aparentemente escindidas derivadas de oraciones de predicado nominal atributivas, sea nominal, sea adjetival, e.g.

5. Lo que es Juan es (un) médico. (Moreno Cabrera 1999: 4261)

6. Lo que es Juan es alto. (Moreno Cabrera 1999: 4262)

Sin embargo, estas oraciones difieren de las oraciones atributivas de predicado nominal, respectivamente Juan es (un) médico. y Juan es alto. por el hecho de que en ellas lo que se predica es la identidad de ser médico o de ser alto con una característica muy distintiva de Juan. Por otro lado, a pesar de que el autor citado menciona las oraciones derivadas de las de predicado nominal atributivas entre las oraciones escindidas, para las mencionadas en (5) y (6) no da variantes focalizadas:

7. ?Es (un) médico lo que Juan es.

8. ?Es alto lo que Juan es.

Mientras la da para la nominal de identidad:

9. Quien es Juan es el médico. (Moreno Cabrera 1999: 4261)

Por lo tanto dudamos si se trata aquí de verdaderas oraciones escindidas, ya que nos parece mucho más probable la interpretación de (5) y (6) como oraciones con el predicativo original topicalizado, cf. polaco Lekarzem to Jan jest. Wysoki to Jan jest (Huszcza 2000). Nótese que una topicalización simple del predicativo, a través del cambio de orden, resultaría en crear oraciones de dudosa gramaticalidad:

10. ?Un médico Juan es.

11. ?Alro Juan es.

El parentesco que hay entre las oraciones de predicado nominal identificativas (especificativas) y las oraciones escindidas se hace más patente cuando nos fijamos en la concordancia del verbo copulativo en cuanto al número y persona. En ambos casos la cópula concuerda obligatoriamente con el elemento post-copular:

12. El gerente soy yo.

13. *El gerente es yo.

14. Quien lo hace soy yo.//Soy yo quien lo hace. (escindidas)

15. *Quien lo hace es yo.//Es yo quien lo hace.

lo que conduce a la hipótesis de que en estas oraciones el elemento post-copular es sujeto, y el pre-copular es predicativo. 
En cuanto a las oraciones de predicado nominal simples, el papel del pronombre como sujeto en estos casos ha sido discutido dentro de varios marcos teóricos (Gutiérrez Ordóñez 1986, Fernández Leborans 1999). No obstante, somos de la opinión de que el análisis de esas oraciones no puede servir como modelo para la descripción de las escindidas, pero inversamente, las conclusiones sacadas de ésta puede tener implicaciones que atañen también a las nominales simples.

Lo que se nota en las escindidas es que la concordancia en cuanto a la persona y número de la cópula se produce siempre con el elemento escindido, cuando este se refiere a la persona (sea pronombre, sea sustantivo) y corresponde al sujeto de la oración original e.g.

16. Soy yo quien lo hizo.

17. Somos nosotros quienes más libros compramos.

18. Son mis padres quienes me han regalado la moto. (Moreno Cabrera 1999: 4255)

Ahora, cuando el sujeto escindido no es personal, la concordancia -esta vez solamente en cuanto al número- es opcional:

19. Son/Es laś ambulancias lo que más ruido hace. (Moreno Cabrera 1999: 4255)

No encontramos datos en la literatura sobre la gramaticalidad de oraciones con el objeto directo no preposicionado plural escindido, pero suponemos que admiten tanto la concordancia, como la falta de concordancia:

20. ?SonEs secretarias bilingües lo que busca la empresa.

21. ?Son/Es las revistas lo que más compro.

En todos los demás casos, el verbo copulativo viene en singular, incluso cuando el elemento escindido es el objeto directo personal y por tanto, preposicionado:

22. Es a mis padres a quienes veo todos los fines de semana.

También es singular cuando el elemento escindido es un objeto preposicional o un adverbial:

23. Es de mis hijos de quien más pienso.

24. Es a las cinco cuando es la fiesta.

A la luz de estos datos nuestra hipótesis preliminar de que el elemento escindido es siempre sujeto del verbo copulativo merece un examen crítico. No cabe duda de que en las oraciones (16-18) es legítimo considerar el elemento escindido como sujeto. Ahora en las oraciones de tipo (19) en la variante con la cópula en plural el elemento escindido sigue siendo sujeto, pero en la variante con la cópula en singular lo es más bien la relativa. Por otro lado, en las oraciones de tipo (22-25) sería difícil considerar como sujeto el elemento escindido preposicionado. Sin embargo nada impide considerar la relativa, incluso encabezada por una preposición, como sujeto, ya que la preposición marca la función sintáctica 
del nexo relativo dentro de la relativa y no influye en el carácter nominal o no de la relativa entera, e.g.

25. Con quien me quiero casar vive a la vuelta. (Brucart 1999:452)

26. A donde va Luis es demasiado frio para pasar las vacaciones. (ibidem)

27. En quien más confiaba me traicionó. (ibidem)

De ahí tenemos que concluir que en el español, al contrario de las lenguas que encabezan el elemento escindido con el pronombre o la proforma, la escisión se produce de dos maneras diferentes. Cuando el elemento escindido es sujeto personal de la original, se vuelve sujeto y la relativa funciona como predicativo. Por otro lado, cuando el elemento escindido está encabezado por la preposición es la relativa la que funciona como sujeto y el elemento escindido vuelve a ser predicativo. En el caso de los sujetos no personales y posiblemente los objetos directos no preposicionados existe una doble opción.

El hecho de que un elemento preposicionado de la original no pueda ser sujeto de la escindida está relacionado con otra característica de la escisión española: El español tiende a conservar relativamente intacta la estructura sintáctica de la oración original. Eso se demuestra por el hecho de que tanto el elemento escindido, como la relativa, tienen que guardar la proposición original, o introducir un nexo relativo equivalente a una construcción preposicional, e.g.

28. *Es Juan a quien veo todos los días.

29. *Es a Juan quien veo todos los dias.

30. Es a las cinco cuando hay la fiesta.

Las estructuras que rompen con esta regla aparecen sólo en el español de América y son generalmente consideradas galicismos (Moreno Cabrera 1999: 4281).

Las observaciones sobre la forma de la cópula en las oraciones escindidas españolas, así como la forma en que aparecen los elementos escindidos en comparación con lo que se puede ver en la literatura en cuanto a otras lenguas nos lleva a una conclusión preliminar de que la escisión no se produce de manera igual en todas las lenguas. Al contrario, existe una tipología de oraciones escindidas en cuando a la "profundidad de escisión", o el grado en que la escisión respecta la estructura sintáctica original. Una vez que hayamos llegado a la conclusión que el elemento nominal (sustantivo o pronombre) con el que concuerda la cópula es su sujeto, podemos estudiar las implicaciones de esta análisis para las oraciones copulativas de identidad especificativas (véase supra). La semejanza entre ellas y las escindidas no se limita a la concordancia sino también abarca la estructura comunicativa. En ambas el elemento post-copular es foco. Compárense una descriptiva y especificativa correspondientes:

31. Yo soy el gerente.

12. El gerente soy yo. 
Consideramos válido decir que en dos tipos de oraciones identificativas lo que cambia no es la estructura sintáctica sino la estructura comunicativa y en las especificativas el elemento post-copular sigue siendo sujeto. Lo que las distingue es que mientras en (31) el sujeto es tópico, en (12) se vuelve foco.

Las peculiaridades de la concordancia verbal en las oraciones escindidas españolas no se limitan al verbo copulativo. También el verbo de la relativa puede adquirir formas diferentes, cuando el elemento escindido es sujeto.

En las oraciones con pronombre personal de primera o segunda persona puede aparecer en la tercera persona, como en (14), pero también en la primera o segunda respectivamente:

32. Soy yo quien lo hago. / Quien lo hago soy yo.

33. Eres tú quien lo haces. // Quien lo haces eres tú.

Este tipo de concordancia se da más bien en el lenguaje coloquial (Moreno Cabrera 1999: 4258).

Cuando el sujeto es plural y personal, el pronombre relativo y por consecuencia el verbo de la relativa tienen que ir en plural, como en (17) y (18).

Ahora, con el sujeto plural no personal hay vacilación, o sea, cuando el verbo copulativo viene en plural, también el verbo de relativa puede ( $\mathrm{y}$ a veces debe) aparecer en plural. La pluralización del nexo relativo se hace entonces obligatoria. Esta concordancia se produce tanto en las escindidas focalizantes, como en las topicalizantes, o sea encabezadas por la relativa, e.g.

34. Son las ambulancias las que más ruido hacen. // Las que más ruido hacen son las ambulancias.

A nuestro parecer, las estructuras concordadas, del tipo (34), en general difieren de las no concordadas, del tipo (19), por su importe informativo. No se debe olvidar que en las estructuras analizadas aquí el contenido de la relativa forma parte del tópico, y el elemento escindido es el foco. La pluralidad gramatical de la relativa hace que la idea de pluralidad sea parte del tópico del cnunciado - de ahí la pluralidad se hace común al tópico y al foco-. Cuando la relativa viene en singular la pluralidad está sólo aportada por el foco. En las escindidas focalizantes el oyente recibe la información sobre la pluralidad a través del elemento escindido; la relativa solamente lo confirma. En las escindidas topicalizantes en singular la idea de la pluralidad sólo se introduce en el foco.

De interés sería entonces estudiar con que motivos posibles el hablante adopta una u otra versión. Una hipótesis digna de averiguar está relacionada con la interpretación del grupo nominal escindido en plural; en concreto con su interpretación como genérica o no. Eso significaría que en las oraciones discordadas, del tipo (19) el grupo nominal las ambulancias se refiriera a las ambulancias en general, en oposición de otros coches. Ahora en las oraciones concordadas, de tipo (34) el hablante se referiría a un conjunto de ambulancias que tanto él, como su 
oyente, serían capaces de identificar. Si es así, mejor se entenderá la dudosa aceptabilidad de:

35. ?Es los trenes lo que ha llegado tarde. (Moreno Cabrera 1999: 4255)

donde el tiempo gramatical de la relativa excluye la interpretación genérica del enunciado.

Más interesantes aún son las oraciones de primera y segunda persona (32-33), en las que el verbo de la relativa no viene en la tercera persona, sino en la primera o segunda respectivamente. En este caso el foco no aporta ninguna información que no haya hecho parte del tópico. La única función informativa que tiene entonces es de confirmar o reforzar la información ya obtenida por el oyente en las topicalizantes y por obtener en las focalizantes. Eso hace pensar que estas estructuras son las únicas con el foco verdaderamente marcado contrastivamente ('yo/tu y no otro', respectivamente). De nuevo, eso sugiere que en las escindidas ordinarias no hay marco de contraste ni sobre el foco, ni sobre el tópico -contrariamente de lo que se supone-generalmente. Otro argumento a favor de tal hipótesis es, que tanto dentro del elemento escindido, como dentro de la relativa, hay posibilidad de marcar contrastivamente un elemento por entonación.

Las últimas observaciones nos conducen a poner una pregunta fundamental sobre el carácter de las oraciones escindidas en español. Dada la abundancia, ante todo de las focalizantes, en los textos escritos y la posibilidad de marcar en ellas los elementos tanto del foco, como del tópico, es de sospechar que la escisión no produce en realidad oraciones de foco o tópico marcado, sino oraciones solamente con la división en tópico y foco netamente marcada.

\section{REFERENCIAS BIBLIOGRÁFICAS}

Brucart, J. M. (1999), La estructura del sintagma nominal: Las oraciones de relativo, en I. Bosque Muñoz, V. Demonte Barreto (eds.), Gramática descriptiva de la lengua española. Madrid: Espasa, 395-522.

Fernández Leborans M. ${ }^{2}$ J. (1999), La predicación: Las oraciones copulativas, en I. Bosque Muñoz, V. Demonte Barreto (eds.), Gramática descriptiva de la lengua española. Madrid: Espasa, 2357-2450.

Fernández Ramirez S. (1951), Gramática española. Madrid: Arco/Libros.

Gutiérrez Ordónez S. (1986), Estructuras ecuativas y ecuacionales, en S. Gutiêrrez Ordóñez, Variaciones sobre la Atribución. León: Universidad de Léon, 45-85.

Moreno Cabrera J. (1999), Las funciones informativas: Las perifrasis de relativo y otras construcciones perifrásticas, en I. Bosque Muñoz, V. Demonte Barreto (eds.), Gramática descriptiva de la lengua española. Madrid: Espasa, 4245-4300. 